\title{
Dietary Choline Reverses Some, but Not AlI, Effects of Folate Deficiency on Neurogenesis and Apoptosis in Fetal Mouse Brain ${ }^{1-3}$
}

\author{
Corneliu N. Craciunescu, ${ }^{4}$ Amy R. Johnson, ${ }^{4}$ and Steven H. Zeisel ${ }^{4,5 *}$ \\ ${ }^{4}$ Department of Nutrition, School of Public Health and School of Medicine, University of North Carolina at Chapel Hill, Chapel Hill, \\ NC, 27599; and ${ }^{5}$ Nutrition Research Institute, University of North Carolina at Chapel Hill, Kannapolis, NC 28081
}

\begin{abstract}
In mice, maternal dietary folate, a cofactor in 1-carbon metabolism, modulates neurogenesis and apoptosis in the fetal brain. Similarly, maternal dietary choline, an important methyl-donor, also influences these processes. Choline and folate are metabolically interrelated, and we determined whether choline supplementation could reverse the effects of folate deficiency on brain development. Timed-pregnant mice were fed control (CT), folate-deficient (FD), or folate-deficient, choline-supplemented (FDCS) AIN-76 diets from d 11 to 17 (E11-17) of pregnancy, and on E17, fetal brains were collected for analysis. Compared with the CT group, the FD group had fewer neural progenitor cells undergoing mitosis in the ventricular zones of the developing mouse brain septum (47\%; $P<0.01)$, hippocampus (29\%; $P<0.01$ ), striatum (34\%; $P<0.01$ ), and anterior and mid-posterior neocortex (33\% in both areas; $P<0.01$ ). In addition, compared with CT, the FD diet almost doubled the rate of apoptosis in the fetal septum and hippocampus $(P<0.01)$. In the FDCS group, the mitosis rates generally were intermediate between those of the CT and FD groups; mitosis rates in the septum and striatum were significantly greater compared with the FD group and were significantly lower than in the CT group only in the septum and neocortex. In the FDCS group, the hippocampal apoptosis rate was significantly lower than in the FD group $(P<0.01)$ and was the same as in the CT group. In the septum, the apotosis rate in the FDCS group was intermediate between the CT and FD groups' rates. These results suggest that neural progenitor cells in fetal forebrain are sensitive to maternal dietary folate during late gestation and that choline supplementation can modify some, but not all, of these effects. J. Nutr. 140: 1162-1166, 2010.
\end{abstract}

\section{Introduction}

Normal development of fetal brain and spinal cord depends on nutrients derived from the maternal diet. For example, in rodents, maternal diets deficient in folate (1) or choline (2) result in decreased neurogenesis and increased apoptosis in fetal brain. In humans, maternal dietary supplementation with folic acid in the periconceptional period significantly reduces the risk of neural tube defects (3-5). Folate plays a central role in DNA synthesis through de novo purine and thymidine biosynthesis necessary for mitotic cell division, and folate is important in the transfer of methyl groups (6). Choline is needed for the biosynthesis of cell membranes as a methyl-group donor and for cholinergic neurotransmission (7). Folate is interrelated metabolically to choline; both methyltetrahydrofolate and

\footnotetext{
${ }^{1}$ Supported by grants from the NIH (DK55865 and AG09525; also DK56350 to the University of North Carolina Nutrition and Obesity Research Center).

${ }^{2}$ Author disclosures: C. N. Craciunescu, A. R. Johnson, and S. H. Zeisel, no conflicts of interest.

${ }^{3}$ Supplemental Methods for immunohistochemical assessment of mitosis and Image analysis, as well as Supplemental Figures 1 and 2, are available with the online posting of this paper at jn.nutrition.org.

* To whom correspondence should be addressed. E-mail: steven_zeisel@unc. edu
}

betaine (derived from choline) can methylate homocysteine to produce methionine (7-11).

Dietary folate intake during pregnancy has been an area of focus for nutrition research $(3,6,12-14)$, and to reduce the incidence of neural tube defects, the food supply in the US has been fortified with folic acid. Pregnancy and lactation are times when demand for choline is especially high, because transport of choline from mother to fetus depletes maternal choline stores $(15,16)$. The National Academy of Sciences set an adequate intake level for choline (17), but, in the US, $<15 \%$ of pregnant women consume the recommended amount (18). In fact, women in California vary enough in dietary choline intake (from $<300$ to $>500 \mathrm{mg} / \mathrm{d}$ ) to influence the risk that they will have a baby with a birth defect; at least $25 \%$ of women consume so little choline that their pregnancies are at 4-fold increased risk $(19,20)$.

Previous studies examined the effects of folate or choline on brain development separately and did not determine whether choline could substitute for folate in the diet. In this study, we tested the hypothesis that supplementation of maternal diets with choline can mitigate the negative effects of folate deficiency on neurogenesis in developing mouse brain. 


\section{Methods}

Mice and diets. Timed-pregnant C57Bl/6J mice were from Jackson Laboratory and were housed individually in cages in a temperaturecontrolled room at $24^{\circ} \mathrm{C}$ and exposed to a 12-h-light and -dark cycle. Mice were placed in cages that contained wire mesh flooring that separated the mice from their feces to avoid coprophagy, a major source of folic acid (21). The control diet group was housed on normal rodent bedding. Mice consumed an AIN-76A pelleted diet (Dyets) (22) with the standard 1.1-g choline chloride/kg diet, $2 \mathrm{mg}$ folic acid/kg diet (22), and $1 \%$ succinyl sulfathiazole (kills intestinal bacteria able to synthesize folate) (23). Pregnant mice were permitted ad libitum access to diet and water until the end of $\mathrm{d} 11$ of gestation when they were randomly assigned to 1 of 3 treatment groups: folate deficient (FD; ${ }^{6} \mathrm{AIN}-76 \mathrm{~A}$ diet with $0.0 \mathrm{mg}$ folic acid $/ \mathrm{kg}$ diet, $1.1 \mathrm{~g}$ choline chloride $/ \mathrm{kg}$ diet, and $1 \%$ succinyl sulfathiazole), control (CT), or folate deficient, choline supplemented (FDCS; AIN-76A diet with $0.0 \mathrm{mg}$ folic acid/kg diet, $4.95 \mathrm{~g}$ choline chloride $/ \mathrm{kg}$ diet and $1 \%$ succinyl sulfathiazole). These diets were ingested until the dams (9-11/group) were killed on gestational d 17 (E17). All mouse procedures were approved by the University of North Carolina Institutional Animal Care and Use Committee.

Tissue collection. Fetal brains were collected using the modified technique of Park et al. (24). Pregnant dams from the 3 treatment groups were anesthetized with an intraperitoneal injection of ketamine (200 $\mathrm{mg} / \mathrm{kg}$ body weight) and xylazine ( $20 \mathrm{mg} / \mathrm{kg}$ body weight; Henry Schein). The terminal surgery and tissue collection was performed as described elsewhere (1). Two male fetal brains from each dam were embedded in paraffin and $5-\mu \mathrm{m}$ coronal serial sections containing the brain regions of interest such as the septum, hippocampus, and cortex were cut and applied to glass slides for histological and immunohistochemical assays. Because there is a posterior to anterior gradient of neurogenesis in fetal mouse brain, the sections were reviewed at the time of immunostaining to ensure that they included anatomically reproducible areas of the septum and hippocampus as defined by a standard atlas of the developing brain (25).

Assessment of mitosis. Coronal brain sections were probed with a rabbit anti-phospho-histone H3 (Ser10), Mitosis Marker polyclonal antibody (catalog no. 06-570, Millipore) that recognizes phosphorylated histone $\mathrm{H} 3$, the core protein of the nucleosome, which becomes phosphorylated at the end of prophase, an event that is essential for the maintenance of mitosis-associated chromosome condensation (26). Slides were prepared for autofluorescence reduction and antigen retrieval (27) and histone H3 phosphorylation was assessed as previously described (2) (Supplemental Methods). Sections were mounted using $80 \%$ Tris-buffered glycerol, $\mathrm{pH} 7.0(28)$ and a no. 1 thickness coverglass.

The incidence of phospho-histone H3-labeled cells was measured at the ventricular surface of the ventricular zone (vz) beginning at the junction (septal fork, nucleus accumbens) of the septum (spt) and caudate putamen striatum (str) and extending toward both the cingulated cortex and the frontal (anterior) neocortical ventricular zones (ncx), within the fetal mouse telencephalic portion of the septum, and at the vz surface beginning at the junction of the hippocampus and choroid plexus [hippocampal wedge (29)], and extending toward and over the mid-posterior cortical vz (Supplemental Fig. 1). Cells were counted hemilaterally in 3-6 consecutive serial sections and the values were averaged to obtain a single value/section (region)/fetus. The length of the ventricular zone was nearly identical across diet groups for a given region of brain.

\footnotetext{
${ }^{6}$ Abbreviations used: CDKI, cyclin-dependent kinase inhibitors, p15lnk4B and p27Kip1; CT, control diet; E\#, embryonic (gestation) day \#; FD, folate-deficient diet; FDCS, folate-deficient, choline-supplemented diet; ncx, ventricular zone corresponding to anterior (in septum sections), mid-posterior (in hippocampus sections) neocortex; spt, ventricular zone of the developing septum; str, ventricular zone corresponding to caudate putamen-striatum; vz, ventricular zone.
}

Assessment of apoptosis. Active caspase-3 immunoreactivity was used to detect apoptotic cells in fetal septum and hippocampus. The pretreatment of slides for deparaffinization, antigen retrieval, and blocking was identical to the one for mitosis assessment and was followed by overnight incubation with a primary antibody to cleaved (active) caspase-3 (Asp175) (catalog no. 9664, Cell Signaling Technology) $1 \mathrm{ng} / \mathrm{L}$ in blocking buffer. Then, goat Cy3-anti-rabbit IgG (catalog no. AP132C, Millipore) at 1:500 dilution was applied for $2 \mathrm{~h}$ at room temperature to display the active caspase-3. 4',6-Diamidino-2-phenylindole, nuclear DNA staining $(0.1 \mathrm{ng} / \mathrm{L})$ for $20 \mathrm{~min}$ was used to counterstain nuclear DNA. The active caspase-3 positive cells were identified and scored by a trained observer who did not know the animals' grouping, based upon the presence of yellow fluorescent staining for activated caspase-3. Stained nuclei were usually, but not always, condensed and intense blue fluorescent chromatin was often visible inside, consistent with fragmented DNA. The apoptotic indices for the fetal mouse brain hippocampus of the different groups are presented as the number of apoptotic cells/septal or hippocampal section. Apoptosis was assessed hemilaterally at a final magnification of $200 \times(2,30)$ in $6-12$ septal and hippocampal hemispheres from 3-6 coronal serial sections that were averaged to obtain a single value/ hippocampal section/fetus. The area of the regions of interest was nearly identical across diet groups for a given region of brain.

Statistical analysis. Data are presented as means \pm SEM. All data were analyzed by ANOVA with the diet as the main effect tested. For all tests, 3-6 consecutive sections/brain were used to calculate a single brain's mean and 8-11 mice were used to calculate the group means; each fetus was generated from a different dam. Once it was established that data variances were equal among all groups (using Tests that the Variances are Equal from JMP version 2, SAS), post hoc analysis was done using the Tukey-Kramer Honest Significant Difference test. Means without a common letter differ, $P<0.01$, as indicated in the figures (JMP version 2, SAS).

\section{Results}

Mitosis. Compared with the CT group, maternal folate deficiency decreased the number of neural progenitor cells undergoing cell replication in the ventricular zones of the developing mouse brain septum $(47 \% ; P<0.01)$, striatum (34\%; $P<0.01)$, anterior neocortex (33\%; $P<0.01)$ (Fig. $1 A)$, hippocampus $(29 \% ; P<0.01)$, and mid-posterior neocortex (33\%; $P<0.01$ ) (Fig. 1B). Choline supplementation (FDCS group) restored the mitosis rate to the control rate in striatum but not in the anterior neocortex. In all other brain regions, the rates of mitosis in the FDCS group were intermediate and did not differ from the CT or FD groups.

Apoptosis. On E17, we observed apoptotic cells in the developing septum and hippocampus (as assessed using active caspase 3 immunoreactivity) (Supplemental Fig. 2). The number of apoptotic cells was $96 \%$ greater in FD fetal septum (Fig. $2 A$ ) $(P<0.01)$ and $114 \%$ greater in FD fetal hippocampus $(P<$ 0.01) (Fig. 2B) compared with the CT. Choline supplementation (FDCS group) restored apoptosis rates to the control level in the hippocampus (Fig. 2B), but in the septum, the rate was intermediate to the FD and CT groups and did not significantly differ from either (Fig. 2A).

\section{Discussion}

Much of the interest in folic acid is based on known effects of this vitamin on neural tube closure. We found that maternal dietary folate intake has effects much later in brain development when the hippocampus, septum, and cortex are forming and 


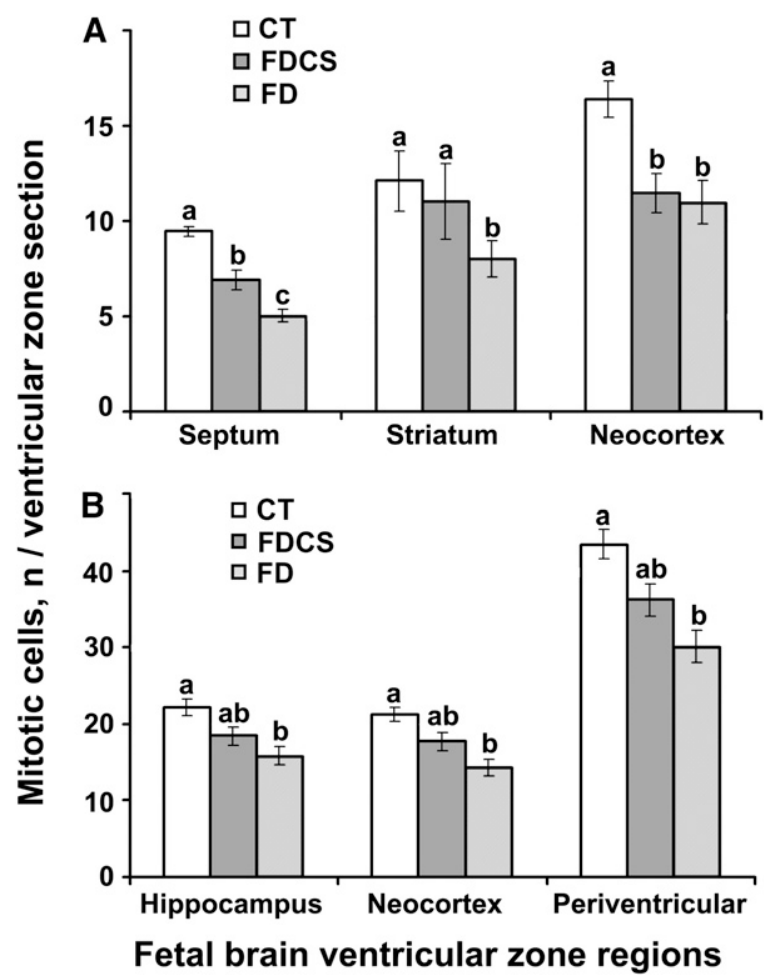

FIGURE 1 Mitotic cells in the forebrain of fetal mice on E17 from timed-pregnant dams fed a CT, FDCS, or FD diet beginning on E11. The $\mathrm{vz}$ regions included the septum, striatum-caudate putamen, anterior neocortex $(A)$, hippocampus, mid-posterior neocortex, and the periventricular region surrounding the lateral ventricle $(B)$. Values are the mean number of phospho-histone $\mathrm{H} 3$ positive cells $\pm \mathrm{SE}, n=8-11$ (means of 3-6 consecutive sections/fetal brain, each from a different dam). For each brain region, means without a common letter differ, $P<0.01$.

when neurogenesis occurs within 2 relatively thin layers of tissue lining the primitive ventricular cavities, referred to as the ventricular zone (vz) and the subventricular zone (31). Maternal folate deficiency was associated with a marked decline in proliferation of neural progenitor cells (Fig. 1) and with a marked increase of apoptotic cell death in these regions (Fig. 2). This is consistent with our previously published results for septum (1). These effects of maternal folate deficiency on fetal brain development are similar to those described when dams are fed a choline-deficient diet during the same embryonic period in rats $(30,32)$ or mice $(2)$.

Mitosis in brain progenitor cells is regulated by cyclindependent kinase inhibitors (CDKI) (33). In previous studies, we showed that feeding pregnant dams a choline-deficient diet increased, whereas a choline-supplemented diet decreased, the expression of CDKI in fetal brain, correlating with reciprocal changes in mitotic cell division by progenitor cells in the vz (34). These changes were mediated in part by epigenetic regulation of the expression of CDKI genes $(35,36)$.

Apoptosis is a regulated form of cell suicide (37) that is important in normal brain development (38-41) and in neurons is modulated by survival factors such as neurotrophins, sex hormones, and neuronal activity $(42,43)$ and is induced by choline deficiency (44-47). We have extensively characterized the choline-regulated intermediate signals that mediate apoptosis $(44,47-50)$ and others have demonstrated that folate deficiency shares these apoptosis pathways $(1,51-53)$. It is reasonable to suggest that choline deficiency and folate defi-

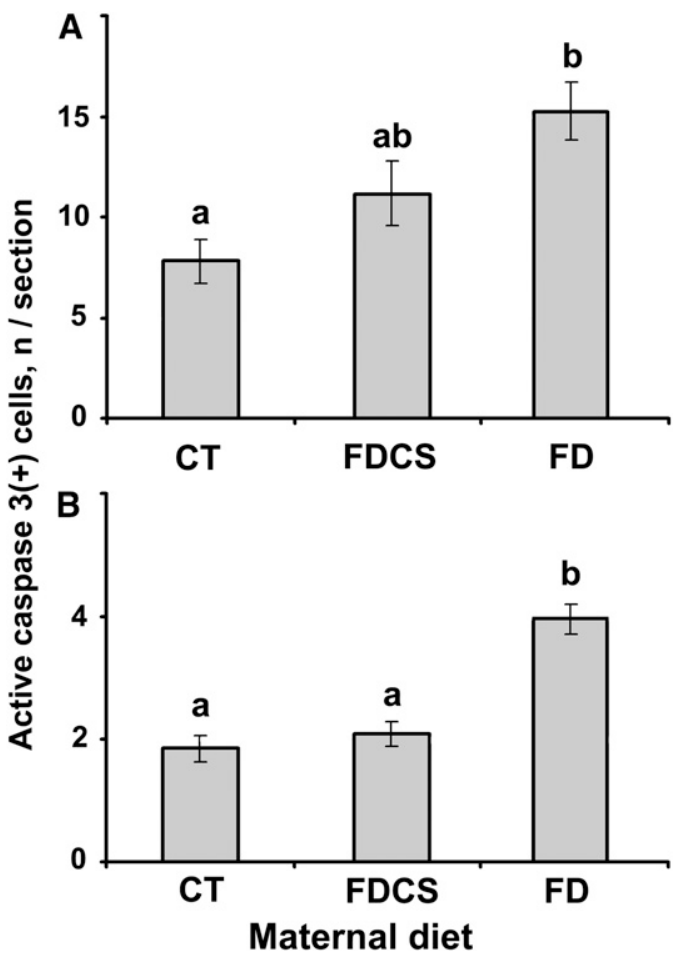

FIGURE 2 Apoptotic neural cells in the septum $(A)$ and hippocampus $(B)$ of fetal mice on E17 from timed-pregnant dams fed a CT, FDCS, or FD diet beginning on E11. Values are the mean number of active caspase 3 positive cells per section $\pm \mathrm{SE}, n=8-11$ (means of 3-6 consecutive sections/fetal brain, each from a different dam). For each brain region, means without a common letter differ, $P<0.01$.

ciency share some common underlying mechanisms that explain the effects on neurogenesis and apoptosis in fetal brain. However, there are folate-specific mechanisms that are not shared with choline; during the S-phase of the cell cycle, folateactivated 1-carbon groups are required for the de novo synthesis of purines (requires 10-formyl-THF for the C2 and C8 carbons of the purine ring) and thymidylate (requires methylene-THF for the reductive methylation of dUMP to form dTMP) (54).

As noted earlier, the metabolism of choline, homocysteine, and methyl-folate are closely interrelated. Perturbing metabolism of one of the methyl-donors results in compensatory changes in the other methyl-donors due to the intermingling of these metabolic pathways (8-10). We found that supplementing folate-deficient mouse dams with choline partially mitigated the effects of folate deficiency on fetal brain neural progenitor cell mitosis and apoptosis (Figs. 1 and 2), but the extent of this mitigation varied in different regions of brain. In some areas (e.g. striatum), mitotic rates were the same as in the CT, whereas in other areas, mitotic rates in the supplemented group were intermediate between the CT and FD groups. In the fetal hippocampus, apoptosis rates were restored to control levels in the choline-supplemented group, whereas in the septum, rates were between those of the CT and FD groups. These differences may occur because the timing of regional development in brain proceeds from posterior to anterior and neurogenesis peaks at different times in each region.

There are a number of potential mechanisms whereby choline supplements might mitigate the effects of folate deficiency. Because the pathways of folate and choline metabolism intersect, folate deficiency also depletes concentrations of choline or choline metabolites in tissues (9). If these metabolites influence 
neurogenesis and apoptosis, correcting the concentrations of these metabolites might normalize brain development. Choline and folate are both important for methyl-metabolism and dietary manipulation of both can alter methylation reactions, including methylation of DNA and histones important for the epigenetic control of gene expression $(36,55-57)$. It is possible that the effects of both nutrients on brain development are mediated by epigenetic events (58).

To date, the majority of scientific investigations about dietary folate requirements during pregnancy focused on folate's role in preventing neural tube defects. This has led to recommendations that pregnant women take supplemental folic acid before and during the first weeks of pregnancy (59). The data presented here suggest that folate availability affects brain development long after neural tube closure and indicates that it may be very important that women have adequate intakes of folic acid throughout pregnancy. Dietary choline status also is important for brain development and we have now found that choline can moderate some of the effects of folate deficiency. This important interaction should be considered as new dietary recommendations for both nutrients during pregnancy are developed.

\section{Acknowledgments}

S.H.Z. and C.N.C. designed the research; C.N.C and A.R.J. conducted research; S.H.Z. and C.N.C. analyzed data; all authors wrote the paper. S.H.Z. had primary responsibility for final content. All authors read and approved the final manuscript.

\section{Literature Cited}

1. Craciunescu CN, Brown EC, Mar MH, Albright CD, Nadeau MR, Zeisel SH. Folic acid deficiency during late gestation decreases progenitor cell proliferation and increases apoptosis in fetal mouse brain. J Nutr. 2004;134:162-6.

2. Craciunescu CN, Albright CD, Mar MH, Song J, Zeisel SH. Choline availability during embryonic development alters progenitor cell mitosis in developing mouse hippocampus. J Nutr. 2003;133:3614-8.

3. Daly LE, Kirke PN, Molloy A, Weir DG, Scott JM. Folate levels and neural tube defects. Implications for prevention. JAMA. 1995;274:1698-702.

4. Czeizel AE, Dudas I. Prevention of the first occurrence of neural-tube defects by periconceptional vitamin supplementation. N Engl J Med. 1992;327:1832-5.

5. Moyers S, Bailey LB. Fetal malformations and folate metabolism: review of recent evidence. Nutr Rev. 2001;59:215-24.

6. Bailey LB, Gregory JF III. Folate metabolism and requirements. J Nutr. 1999;129:779-82.

7. Zeisel SH, Blusztajn JK. Choline and human nutrition. Annu Rev Nutr. 1994;14:269-96.

8. Varela-Moreiras G, Selhub J, da Costa K, Zeisel SH. Effect of chronic choline deficiency in rats on liver folate content and distribution. J Nutr Biochem. 1992;3:519-22.

9. Kim YI, Miller JW, da Costa KA, Nadeau M, Smith D, Selhub J, Zeisel $\mathrm{SH}$, Mason JB. Severe folate deficiency causes secondary depletion of choline and phosphocholine in liver. J Nutr. 1994;124:2197-203.

10. Selhub J, Seyoum E, Pomfret EA, Zeisel SH. Effects of choline deficiency and methotrexate treatment upon liver folate content and distribution. Cancer Res. 1991;51:16-21.

11. Schwahn BC, Chen Z, Laryea MD, Wendel U, Lussier-Cacan S, Genest J $\mathrm{Jr}$, Mar MH, Zeisel SH, Castro C, et al. Homocysteine-betaine interactions in a murine model of 5,10-methylenetetrahydrofolate reductase deficiency. FASEB J. 2003;17:512-4.

12. Beaudin AE, Stover PJ. Folate-mediated one-carbon metabolism and neural tube defects: balancing genome synthesis and gene expression. Birth Defects Res C Embryo Today. 2007;81:183-203.

13. O'Connor DL. Folate status during pregnancy and lactation. Adv Exp Med Biol. 1994;352:157-72.
14. van Rooij IA, Vermeij-Keers C, Kluijtmans LA, Ocke MC, Zielhuis GA, Goorhuis-Brouwer SM, van der Biezen JJ, Kuijpers-Jagtman AM, Steegers-Theunissen RP. Does the interaction between maternal folate intake and the methylenetetrahydrofolate reductase polymorphisms affect the risk of cleft lip with or without cleft palate? Am J Epidemiol. 2003;157:583-91.

15. McMahon KE, Farrell PM. Measurement of free choline concentrations in maternal and neonatal blood by micropyrolysis gas chromatography. Clin Chim Acta. 1985;149:1-12.

16. Zeisel SH, Mar M-H, Zhou Z-W, da Costa K-A. Pregnancy and lactation are associated with diminished concentrations of choline and its metabolites in rat liver. J Nutr. 1995;125:3049-54.

17. Institute of Medicine, National Academy of Sciences USA. Choline. Dietary reference intakes for folate, thiamin, riboflavin, niacin, vitamin B12, panthothenic acid, biotin, and choline. Washington, DC: National Academy Press; 1998. p. 390-422.

18. Jensen HH, Batres-Marquez SP, Carriquiry A, Schalinske KL. Choline in the diets of the U.S. population: NHANES, 2003-2004. FASEB J. 2007;21:lb219.

19. Shaw GM, Carmichael SL, Yang W, Selvin S, Schaffer DM. Periconceptional dietary intake of choline and betaine and neural tube defects in offspring. Am J Epidemiol. 2004;160:102-9.

20. Shaw GM, Carmichael SL, Laurent C, Rasmussen SA. Maternal nutrient intakes and risk of orofacial clefts. Epidemiology. 2006;17: 285-91.

21. Ebino KY, Yoshinaga K, Suwa T, Kuwabara Y, Takahashi KW. Effects of prevention of coprophagy on pregnant mice-is coprophagy beneficial on a balanced diet? Jikken Dobutsu. 1989;38:245-52.

22. AIN. Report of the American Institute of Nutrition ad hoc committee on standards for nutritional studies. J Nutr. 1977;107:1340-8.

23. Said HM, Chatterjee N, Haq RU, Subramanian VS, Ortiz A, Matherly LH, Sirotnak FM, Halsted C, Rubin SA. Adaptive regulation of intestinal folate uptake: effect of dietary folate deficiency. Am J Physiol Cell Physiol. 2000;279:C1889-95.

24. Park JJ, Baum MJ, Paredes RG, Tobet SA. Neurogenesis and cell migration into the sexually dimorphic preoptic area/anterior hypothalamus of the fetal ferret. J Neurobiol. 1996;30:315-28.

25. Jacobowitz DM, Abbott LC. Chemoarchitectonic atlas of the developing mouse brain. Boca Raton (FL): CRC Press; 1998.

26. Hans F, Dimitrov S. Histone H3 phosphorylation and cell division. Oncogene. 2001;20:3021-7.

27. Baschong W, Suetterlin R, Laeng RH. Control of autofluorescence of archival formaldehyde-fixed, paraffin-embedded tissue in confocal laser scanning microscopy (CLSM). J Histochem Cytochem. 2001;49:1565-72.

28. Romijn HJ, van Uum JF, Breedijk I, Emmering J, Radu I, Pool CW. Double immunolabeling of neuropeptides in the human hypothalamus as analyzed by confocal laser scanning fluorescence microscopy. J Histochem Cytochem. 1999;47:229-36.

29. Altman J, Bayer SA. Mosaic organization of the hippocampal neuroepithelium and the multiple germinal sources of dentate granule cells. J Comp Neurol. 1990;301:325-42.

30. Albright CD, Friedrich CB, Brown EC, Mar MH, Zeisel SH. Maternal dietary choline availability alters mitosis, apoptosis and the localization of TOAD-64 protein in the developing fetal rat septum. Brain Res Dev Brain Res. 1999;115:123-9.

31. The Boulder Committee. Embryonic vertebrate central nervous system: revised terminology. The Boulder Committee. Anat Rec. 1970;166:257-61.

32. Albright CD, Tsai AY, Friedrich CB, Mar MH, Zeisel SH. Choline availability alters embryonic development of the hippocampus and septum in the rat. Brain Res Dev Brain Res. 1999;113:13-20.

33. Leshem Y, Halevy O. Phosphorylation of $\mathrm{pRb}$ is required for HGFinduced muscle cell proliferation and is p27kip1-dependent. J Cell Physiol. 2002;191:173-82.

34. Albright CD, Mar MH, Friedrich CB, Brown EC, Zeisel SH. Maternal choline availability alters the localization of p15Ink4B and p27Kip1 cyclin-dependent kinase inhibitors in the developing fetal rat brain hippocampus. Dev Neurosci. 2001;23:100-6.

35. Niculescu MD, Craciunescu CN, Zeisel SH. Gene expression profiling of choline-deprived neural precursor cells isolated from mouse brain. Brain Res Mol Brain Res. 2005;134:309-22.

36. Niculescu MD, Craciunescu CN, Zeisel SH. Dietary choline deficiency alters global and gene-specific DNA methylation in the developing hippocampus of mouse fetal brains. FASEB J. 2006;20:43-9. 
37. Schwartzman RA, Cidlowski JA. Apoptosis: the biochemistry and molecular biology of programmed cell death. Endocr Rev. 1993;14: 133-51.

38. Ferrer I. Cell death in the normal developing brain, and following ionizing radiation, methyl-azoxymethanol acetate, and hypoxia-ischaemia in the rat. Neuropathol Appl Neurobiol. 1996;22:489-94.

39. Davis EC, Popper P, Gorski RA. The role of apoptosis in sexual differentiation of the rat sexually dimorphic nucleus of the preoptic area. Brain Res. 1996;734:10-8.

40. Ferrer I, Tortosa A, Blanco R, Martin F, Serrano T, Planas A, Macaya A. Naturally occurring cell death in the developing cerebral cortex of the rat. Evidence of apoptosis-associated internucleosomal DNA fragmentation. Neurosci Lett. 1994;182:77-9.

41. Naruse I, Keino H. Apoptosis in the developing CNS. Prog Neurobiol. 1995;47:135-55.

42. Henderson CE. Programmed cell death in the developing nervous system. Neuron. 1996;17:579-795.

43. Vekrellis K, McCarthy M, Watson A, Whitfield J, Rubin L, Ham J. Bax promotes neuronal cell death and is downregulated during the development of the nervous system. Development. 1997;124: 1239-49.

44. Holmes-McNary MQ, Baldwin AS Jr, Zeisel SH. Opposing regulation of choline deficiency-induced apoptosis by p53 and NF-k\{kappa\}B. J Biol Chem. 2001;276:41197-204.

45. Holmes-McNary MQ, Loy R, Mar MH, Albright CD, Zeisel SH. Apoptosis is induced by choline deficiency in fetal brain and in PC12 cells. Brain Res Dev Brain Res. 1997;101:9-16.

46. Yen CL, Mar MH, Meeker RB, Fernandes A, Zeisel SH. Choline deficiency induces apoptosis in primary cultures of fetal neurons. FASEB J. 2001;15:1704-10.

47. Yen CL, Mar MH, Zeisel SH. Choline deficiency-induced apoptosis in PC12 cells is associated with diminished membrane phosphatidylcholine and sphingomyelin, accumulation of ceramide and diacylglycerol, and activation of a caspase. FASEB J. 1999;13:135-42.

48. Albright CD, Salganik RI, Kaufmann WK, Vrablic AS, Zeisel SH. A p53-dependent G1 checkpoint function is not required for induction of apoptosis by acute choline deficiency in immortalized rat hepatocytes in culture. J Nutr Biochem. 1998;9:476-81.

49. Vrablic AS, Albright CD, Craciunescu CN, Salganik RI, Zeisel SH. Altered mitochondrial function and overgeneration of reactive oxygen species precede the induction of apoptosis by 1-O-octadecyl-2-methylrac-glycero-3-phosphocholine in p53-defective hepatocytes. FASEB J. 2001;15:1739-44.

50. Yen CL, Mar MH, Craciunescu CN, Edwards LJ, Zeisel SH. Deficiency in methionine, tryptophan, isoleucine, or choline induces apoptosis in cultured cells. J Nutr. 2002;132:1840-7.

51. Chern CL, Huang RF, Chen YH, Cheng JT, Liu TZ. Folate deficiency-induced oxidative stress and apoptosis are mediated via homocysteine-dependent overproduction of hydrogen peroxide and enhanced activation of NFkappaB in human Hep G2 cells. Biomed Pharmacother. 2001;55:434-42.

52. Huang RF, Ho YH, Lin HL, Wei JS, Liu TZ. Folate deficiency induces a cell cycle-specific apoptosis in HepG2 cells. J Nutr. 1999;129:25-31.

53. James SJ, Miller BJ, Basnakian AG, Pogribny IP, Pogribna M, Muskhelishvili L. Apoptosis and proliferation under conditions of deoxynucleotide pool imbalance in liver of folate/methyl deficient rats. Carcinogenesis. 1997;18:287-93.

54. Stover PJ. One-carbon metabolism-genome interactions in folateassociated pathologies. J Nutr. 2009;139:2402-5.

55. Waterland RA, Dolinoy DC, Lin JR, Smith CA, Shi X, Tahiliani KG. Maternal methyl supplements increase offspring DNA methylation at Axin fused. Genesis. 2006;44:401-6.

56. Waterland RA, Lin JR, Smith CA, Jirtle RL. Post-weaning diet affects genomic imprinting at the insulin-like growth factor 2 (Igf2) locus. Hum Mol Genet. 2006;15:705-16.

57. Mehedint MG, Niculescu MD, Craciunescu CN, Zeisel SH. Choline deficiency alters global histone methylation and epigenetic marking at the Re1 site of the calbindin 1 gene. FASEB J. 2010;24:184-95.

58. Zeisel SH. Epigenetic mechanisms for nutrition determinants of later health outcomes. Am J Clin Nutr. 2009;89:S1488-93.

59. Food and Nutrition Board Institute of Medicine. Dietary reference intakes: folate, other B vitamins, and choline. Washington, DC: National Academy Press; 1998. 